\title{
LOCALITY AND RESOURCE AWARE PEER-TO-PEER OVERLAY NETWORKS
}

\author{
(Invited)
}

\author{
Chun-Hung Wu, Kuo Chiang, Ruo-Jian Yu, and Sheng-De Wang
}

\begin{abstract}
Unstructured peer-to-peer ( $\mathrm{P} 2 \mathrm{P})$ overlay architectures are attracting more and more attention. In order to solve the topology mismatch problem, many approaches take locality information into account when designing peer-to-peer overlay networks. In this paper, we not only exploit locality but also take resource types into consideration. Taking advantage of data replication, selective search, clustering, and interest groups, we can improve the search performance of unstructured P2P networks. Simulation results show that our algorithm is better than the mOverlay network in the number of messages per search while it maintains almost the same hit ratio and comes with competitive locality properties.
\end{abstract}

Key Words: peer-to-peer networks, overlay networks, locality aware, resource aware.

\section{INTRODUCTION}

Peer-to-peer overlay networks are virtual networks built on top of underlying networks (Doval and O'Mahony, 2003) and can provide various services, such as video streaming and file sharing. Gnutella and Napster are pioneers in peer-to-peer file sharing systems. The two peer-to-peer systems are unstructured, and the allocation of files is completely unrelated with the topology of the overlay networks. They provide a convenient environment to share objects or files among a large number of users. Because of the limits of query flooding and the central index service scheme, respectively, Gnutella and Napster both suffer a scalability problem. Much research has been devoted to develop new locating and searching technologies for peer-to-peer systems. Many peer-to-peer systems take advantage of structured overlay networks, such as distributed hash table (DHT) techniques to distribute objects evenly. Among these, CAN (Ratnasamy et al., 2001), Chord (Stoica et al., 2001), Pastry (Rowstron and Druschel, 2001), and Tapestry (Zhao et al., 2001) are well-known infrastructures that deploy DHT as the fundamental part. Two

*Corresponding author. (Tel: 886-2-33663579; Fax: 886-223671909; Email: sdwang@ntu.edu.tw)

The authous are with the Department of Electrical Engineering, National Taiwan University, Taipei 106, Taiwan, R.O.C. neighbors in virtual overlay networks may actually span two distant countries in underlying networks. Furthermore, according to Lv et al. (2002), when nodes frequently join or leave the overlay network, structured peer-to-peer systems take much time in order to keep the structure of the overlay network. On the other hand, DHT-based structured networks are not well suited for keyword search, although the search performance of structured peer-to-peer systems is more efficient than unstructured peer-to-peer systems.

In unstructured peer-to-peer networks, to reduce the bandwidth requirement and improve the efficiency of the flooding mechanism, two classes of methods are used. The first class of methods is based on selective search including biased search and blind search. Biased search sends messages only to the nodes that are more likely to get the information, instead of transmitting messages to all their neighbors. The methods are used in Yang and Garcia-Molina (2002), Ratnasamy et al. (2002), and Lv et al. (2002). Blind search confines the nodes to transmitting messages to some adjacent nodes instead of all adjacent nodes without using the information of messages or choosing the best path for transmitting. The methods of random walk (Lv et al., 2002) and dynamic query (Xu et al., 2003) belong to blind search. In the second class of methods, nodes make use of caching or replication mechanisms to keep the known objects to enhance the search efficiency. The most 
(a)

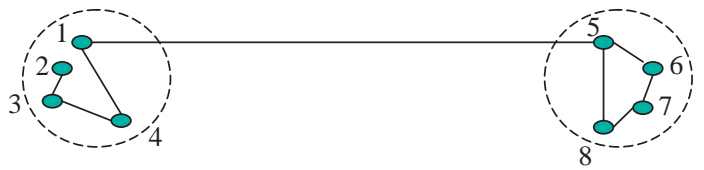

(b)

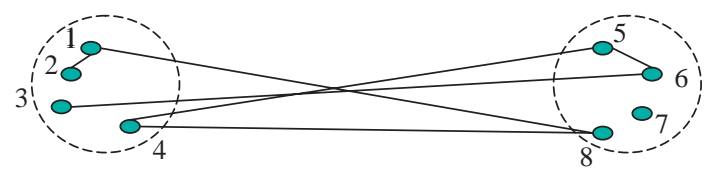

Fig. 1 (a) An overlay network concerning locality and (b) an overlay network built randomly

common way is uniform index caching (UIC), where the locations of files are recorded in caches once the files are successfully found in a process of search, so the files will be found easily later. The method is used by Patro and $\mathrm{Hu}$ (2003), Chiang et al. (2007), Cohen and Shenker (2002), Bestavros and Jin (2003), and Wang and Vanninen (2004). Although the selective search and the caching mechanism can improve the search efficiency of peer-to-peer networks, the topology mismatch problem of the overlay networks still exists.

To further improve the efficiency, locality should be considered by multicast or routing algorithms of the application layer when constructing overlay networks (Zhang et al., 2004), (Zhang et al., 2005), (Kobayashi et al., 2005), (Shamsi et al., 2005), (Sun et al., 2006), (Shin et al., 2002), (Xiao et al., 2005). Two common frameworks are based on the structures of trees and meshes, respectively. For example, the Narada protocol (Chu et al., 2000), which is suitable for small overlay networks, is based on meshes. The NICE application layer multicast protocol (Banerjee et al., 2002) and the host multicast tree protocol (HMTP) (Zhang et al., 2002) are based on the structures of trees. Thus, when nodes are added to the overlay networks, they have to notify the node at the highest level. The highest level node becomes a hotspot; if the hotspot fails, the systems fail. The binning scheme (Ratnasamy et al., 2002) uses a set of nodes called landmarks to consider the locality information and thus improve overall efficiency of the network. Although the method has improved the efficiency, it needs extra information about landmarks and may incur hotspots when the overlay network grows. To construct an overlay network that takes account of locality of peers, the mOverlay network (Zhang et al., 2004) is proposed to decrease the communication cost between nodes. The mOverlay network uses a dynamic landmark technique to achieve the load balance and avoid the hotspots. In this paper, we consider not only the locality and but also the resource types in an overlay similar to the mOverlay network.

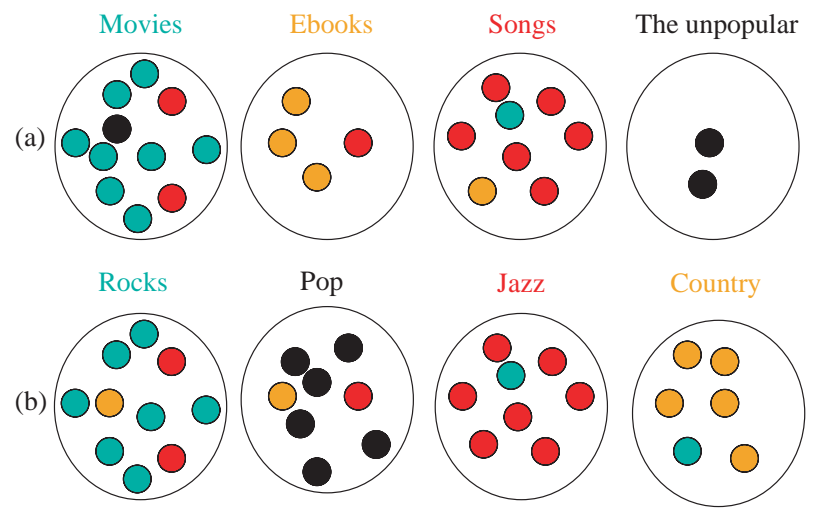

Fig. 2 (a) The groups of resource types and (b) the groups of song types

Locality is also considered in (Rowstron et al., 2001) and (Shamsi et al., (2005). In (Rowstron et al., 2001), the nodes with high hit ratio are put near the nodes that issue queries, but it does not utilize the concept of clustering. In this case, the search would incur a lot of messages transmitted in the network. In (Shamsi et al., 2005), similar files are put in the same semantic overlay, and there are several clusters in a semantic overlay. This mechanism is similar to ours. But in the aspect of locality, in (Shamsi et al., 2005), TTL are assigned to decide which cluster to join in a semantic overlay. Since the distribution of real underlying networks is not considered, it cannot solve the problem of topology mismatch.

\section{OVERLAY NETWORKS WITH THE AWARENESS OF LOCALITY AND RESOURCE TYPES}

The concept of locality can be viewed as the distance between two peers, network latency, round trip time, or the minimum bandwidth between two nodes. To solve the problem of mismatch (Liu et al., 2004), we hope that adjacent nodes in the underlying network are also adjacent in the overlay network. As an example, Fig. 1(a) (Zhang et al., 2004) shows an overlay network with consideration of locality, resulting in less cross-group communications, while Fig. 1 (b) does not take the locality into account, leading to many cross-group communications.

The concept of interest groups (Chen et al, 2006) can play an important role in designing peer-to-peer systems. Everyone has his own interest and the communities in the Internet also reflect the fact. If we can place files with the same attribute or files interesting the same group of users in the same cluster, the users can easily find the files by searching for them within the same cluster. The concept is shown in Fig. 2(a) for general resource types or as Fig. 
2(b) for types of songs. It is worthy of note that the resource types themselves present the properties of fractals both in coarse and fine classifications of resources. The proposed overlay network is a twolevel scheme, called LARO networks, with the concept of grouping by factors of data replication, clustering, and interest groups. Due to the fact that we take both locality and resource types into consideration in our algorithm, there may be some files added to a cluster because of locality, although they do not match the resource type of the cluster.

The proposed LARO overlay networks is a two-level scheme that makes use of the concepts of locality and resource awareness to construct the peerto-peer network. The upper level, level 1, is composed of clusters or groups, and the lower level, level 0 , is composed of nodes. Nodes in the same clusters share some similarity, either locality or resource types. There is a group leader in each cluster. Group leaders manage and keep all the information of the nodes, including peer lists, distances between peers, and resource type number in clusters. Each file in a node belongs to one resource type; for example, the files may be of types of movies, music, or programs. Each node will be assigned a resource type number determined by the majority of resource types of its files. For example, if there are three movie files, one music file, and one program in a node, we assign the node the resource type "movies" as the node's resource type number. And the resource type number of a cluster is the same as the group leader's resource type number. In general, a group leader might be the first node to form the cluster.

To further promote the search efficiency of the proposed peer-to-peer network, we also make use of the concept of data replication. Assuming there is a node with $\mathrm{N}$ files and every file has its resource type number, we do data replication during the locating process that constructs the overlay. Once a node has been added to a group, we randomly select zero to $N$ 1 files and replicate them to the clusters with the same resource type number.

In this paper, clusters or groups are composed of nodes with either the close locations or the same resource type. A locating process is required to help a node find a group to join. We add a node to a group, first considering its resource type and then its locality in the underlying network. This will form a network of groups, with each group mainly comprising the same type of resources. We present the pseudocode of our algorithm as follows.

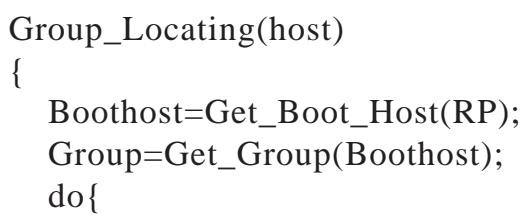

\author{
If(Meet_Resource_Criterion()) \\ return Group; \\ if(Meet_Locality_Criterion(host, Group)) \\ retrun Group; \\ CandidateList=Get_Neighbors(Group); \\ DistanceList=Measure_Distance(Group, \\ CandidateList); \\ (MinDis, MinGroup $)=$ Min $($ DistanceList $)$; \\ Group=MinGroup; \\ if(Meet_Stop_Criterion()) \\ \}while(true) \\ return NewGroup; \\ \}
}

As in the mOverlay network (Zhang et al., 2004), the locating process relies on a node called Rendezvous Point (RP) that has all the information of the overlay network. When a node wants to enter the overlay network, it gets connection with the RP first, and the RP responds to it with a bootstrapping host randomly (Cramer et al., 2004). The method is called dynamic landmark location algorithm (Zhang et al., 2004), which randomly provides the bootstrapping hosts in order to balance the load of nodes in the network. Then, the locating procedure continues to check if the resource type number of the node is the same as that of the cluster that the node wants to join. If they have the same resource type number, we add the node to the cluster directly. If the resource type numbers are different, we consider the cluster's locality. If the distance between the node and the cluster's group leader is less than a threshold value, the node joins the cluster. If the above two conditions are not satisfied, the locating process will enter a recursive procedure. The group and its neighbors will be put in an array and then we find its nearest neighbor considering resource and locality conditions. The recursive process may terminate after a fixed number of trials. In our simulation, we set ten times as our upper bound; namely, the node will form a new cluster and be the leader if the locating procedure occurs over ten times. The node also forms a new cluster if there is no neighbor found in the locating process.

In Fig. 3, we explain the procedure of adding a node to the overlay network. When node $A$ wants to join the overlay network, it first contacts RP for some nodes to try. In each try, node $A$ first checks if the condition of resource types is met and then if the locality condition is satisfied. If yes, node $A$ will be added to the group. If no, we will find the group's nearest neighbor and repeat the same procedure. We can see from Fig. 3 that node $A$ traverses from group 1 to group 4. If group 4 meets the resource or locality conditions, node $A$ is added to group 4 , and the locating process ends.

In the proposed locating process, we first 


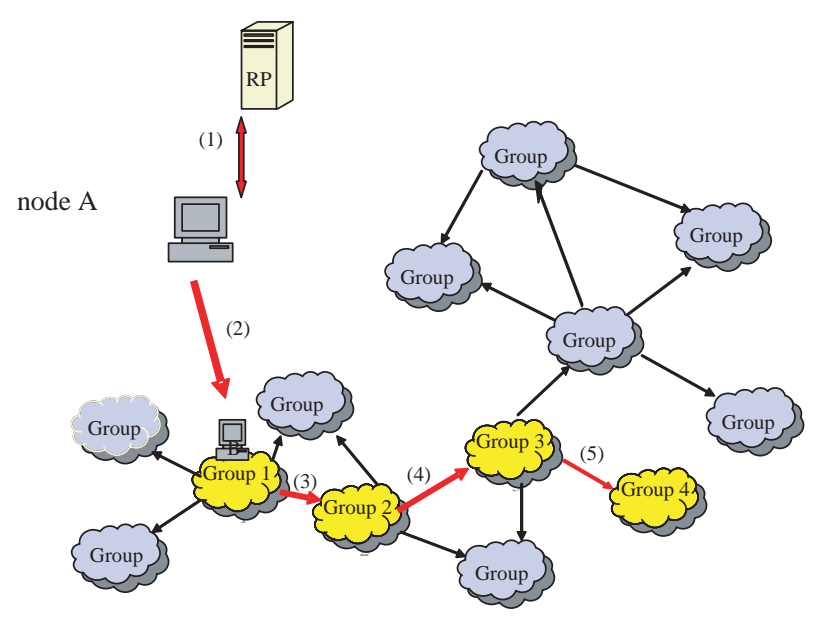

Fig. 3 Locating process

consider the type of resources; if the condition is not satisfied, we will then consider the condition of locality. The problem is that how we set the priority of the two conditions. If we take the condition of distance into consideration first, it may cause lots of files which do not have the same resource type number to be incorporated into clusters, because the nodes are added to a cluster only if their distances are less than a threshold value. In this case, when users search for files in a cluster, they may find too many files with different resource type numbers, making a lot of mismatches in searching. If we take the type of resource into consideration first, the problem is solved. However, there is no perfect strategy. Since if we consider the type of resources first, we will get clusters with longer distances between nodes. In our simulation, we can find that the average distance does not increase that much. So, we decided to first consider the types of resource and then distance.

In summary, we propose using three conditions that make peers join a certain group, namely resource types, locality, and replication. First, a peer's resource type number is the same as the group. Second, the average distance between a peer and the group is smaller than a certain threshold. Third, when a peer joins the group with the same resource type number, it will also randomly select other groups with the same resource type number to join.

There are three conditions to form a new group: first, the first peer enters the overlay network. Second, a peer executes the locating process over a specific number of times. Third, a peer moves to an isolated group with a different resource type number and the average distance between them is over a certain threshold. In forming a new group, there needs to be a new resource type number and $M$ neighbors must be found by running the locating process several times.

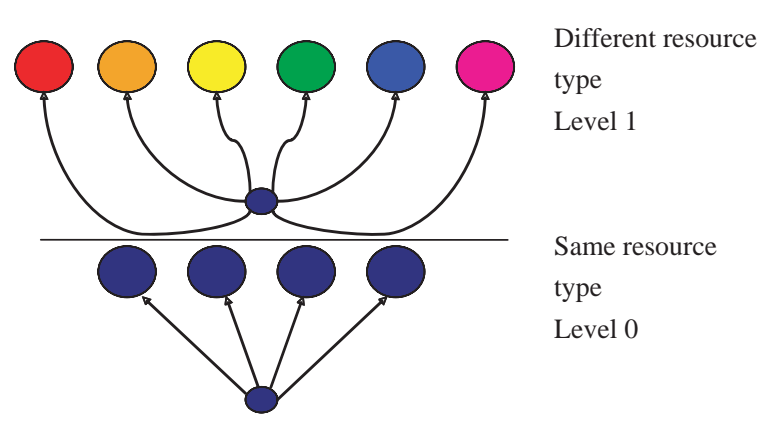

Fig. 4 The proposed two-level random walks

\section{SEARCH ALGORITHMS FOR THE PROPOSED OVERLAY}

\section{Search Algorithms}

The method of selective search is originally used to reduce the requirement of bandwidth and the number of messages during flooding. In this paper, the proposed search algorithm is based on the random walk algorithm (Kobayashi et al., 2005). Because our overlay architecture is a two-level framework, common searching algorithms cannot satisfy our needs. The original random walk algorithm uses a boot host to send a fixed number of walkers to start the search in parallel. It randomly selects the next peers to send the query with each peer having the same probability to be selected. Since our algorithm is based on a two-level architecture as shown in Fig. 4, the proposed random walk algorithm first takes a fixed number of walks in the first level, level 0 and then takes a another fixed number of walks in the second level, level 1. The groups in the first level, level 0 , have the same resource type number as the file; the groups in the second level, level 1, contain those groups with different resource type numbers. For example, if we want to search for a song, first of all, we will search among Song groups, which are in the first level, level 0 . If we cannot find the required song among them, we will search among other groups which are in the second level, level 1.

As an example, we take an $\mathrm{F}$-walker random walk and set $F=10$. When a peer wants to search for a file, it will release 10 walks to groups in the first level. We randomly select a boot host in a group in each walk and randomly take two walks. The search algorithm will stop if we find the required file at this step; if not, a peer will release another 10 walks to groups in the second level, and each walk randomly selects a boot host in a group and randomly takes two steps.

We can view an overlay network as a graph. Each node in a graph represents a group or a peer in the overlay network, and two nodes connected by 
links are neighbors. Assume that an overlay network with $N$ total nodes and each node has $M$ neighbors on average; in other words, each node has $M$ out degrees on average. The nearest group can be found in an overlay network by looking at nodes and edges in a graph. The proof of the following formula about average distance in hops, $d$, between two nodes can be found in (Zhang et al., 2004):

$$
d<\log _{M} N+3
$$

Thus, the average distance in hops of two nodes is $O\left(\log _{M} N\right)$; therefore, a node can find its nearest group in $O(\log N)$ communications.

Connectedness of nodes or connectedness of groups is an important issue in a network ( $\mathrm{Si}$ and $\mathrm{Li}$, 2005). A query in a peer-to-peer network might fail if there are isolated nodes in overlay networks; in other words, we cannot find a path from a start node which is an isolated node to any other groups. To solve this problem, the proposed LARO algorithm will find some groups as neighbor groups when forming a new group. Therefore, it will reduce the probability of forming a disconnected graph.

As compared to mesh-based and tree-based architectures, the proposed two-level scheme is much more efficient to maintain. For an $N$-node peer-topeer network, mesh-based overlay networks (Hsiao and Liao, 2006) suffer from an $O\left(N^{2}\right)$ communication load between two peers. For tree-based overlay networks, the tree root or top-level nodes would be overloaded when peers make queries in this overlay network frequently.

\section{Average Distance Analysis}

We are interested in knowing the average distance for the LARO networks. We assume that there are $N$ groups under the LARO network. Each group, on average contains $n$ nodes and has $M$ group neighbors. Each node in a group has neighbors on average. We use $D_{o}$ to represent the average distance between two groups, and $D_{i}$ to represent the average distance between two nodes in a group. Then, we know there are $N \cdot n \cdot m / 2$ connections inside groups and $N \cdot M / 2$ links between groups. We can get the average distance $\bar{D}$ under LARO networks, the procedure being the same as for mOverlay (Zhang et al., 2004):

$$
\bar{D}=\frac{D_{i} \frac{N \cdot n \cdot m}{2}+D_{o} \frac{N \cdot M}{2}}{\frac{N \cdot n \cdot m}{2}+\frac{N \cdot M}{2}}=\frac{D_{i} \cdot n \cdot m+D_{o} \cdot M}{n \cdot m+M}
$$

Assuming that the intra group node degree $m$ and inter group degree $M$ is related by $m=\lambda M$, we can get

$$
\bar{D}=\frac{D_{i} \cdot \lambda \cdot n+D_{o}}{\lambda \cdot n+1}
$$

Furthermore, by assuming $\lambda n \gg 1$, we have

$$
\bar{D} \cong D_{i}
$$

From Eq. (3), we can see that in the LARO overlay the average distance is close to the intra group distance $D_{i}$ under some conditions. This is reasonable because the conditions $\lambda n \gg 1$ can easily be met by increasing the intra group degree factor, $\lambda$, and maintaining an appropriate average number of nodes in groups, $n$.

On the other hand, we would like to compare the average intra group distances between LARO and mOverlay. Assume the average intra- and inter-group distances of mOverlay are $D_{i n}$ and $D_{o u}$, respectively. It has been shown in Zhang et al. (2004) that if the number of group $N$ satisfies $N>1$, the average distance of a randomly connected overlay, $D_{\text {rco }}$ can be approximated as:

$$
D_{\text {rco }} \cong D_{o u}
$$

In a group of the LARO network, we assume the ratio of the number of peers that have the same resource type number but do not satisfy the locality criterion to the number of peers that meet the locality criterion is $r / s$. Thus, for the intra group distance $D_{i}$ in LARO, we have

$$
D_{i}=\left(r \cdot D_{o u}+s \cdot D_{i n}\right) /(r+s) .
$$

From Eq. (5), it is obvious that

$$
D_{\text {in }}<D_{i}<D_{\text {ou }}
$$

If $r=0$, that is, in any group of LARO, there is no peer that does not meet the locality criterion, the LARO network would reduce to an mOverlay network. Thus

$$
D_{i}=D_{\text {in }} \text { if } r=0
$$

The result shows that if $r \ll s$, the intra group distance of groups of LARO would be close to that of mOverlay.

\section{Discussion}

In this paper, we propose applying the grouping concept to unstructured P2P networks. It is noted that the grouping concept can also be applied to the Distributed hash tables (DHT) based P2P systems such as CAN and Chord. Although DHT-based networks provide efficient platforms with guaranteed searching 
performance, the locality is destroyed since DHTs use a uniform hash function to evenly distribute nodes and objects in the virtual space. The hierarchical architectures based on DHTs, such as HIERAS (Xu et al., 2003) and Canon (Ganesan et al., 2004), can significantly improve locality by grouping nodes geographically. HIERAS combines a hierarchical structure with DHT based routing algorithms like Chord. Besides the biggest ring, it groups topologically adjacent nodes into smaller rings. In a two-layer HIERAS system, for example, $P$ is the Layer- 1 ring which may contain three Layer-2 rings. The nodes within a ring in the lower layer are more adjacent topologically. HIERAS uses the Distributed Binning Scheme (Ratnasamy et al., 2002) to determine which ring the node belongs to. A set of landmark nodes is chosen for dividing a system into disjoint bins. When a node joins HIERAS, it measures distance between landmark nodes and itself. Nodes that get the same order are organized into a smaller ring. In the smaller ring, nodes create proper links and table entries according to the system's DHT structure. Compared to Grapes, HIERAS takes the landmark scheme which adds only extra tables and links in each hierarchical layer but not independent DHTs. Therefore the loads of the nodes in HIERAS are all the same because the consistent hashing produces good loadbalancing. When a node requests an object, it looks up the smallest ring's table first. This method exploits locality because the nodes in the smaller ring are closer than in the bigger ring. HIERAS can reduce hops on DHT based systems significantly, too.

Canon proposes a generic architecture that is based on DHTs with hierarchical structures. It inherits load-balancing offered by a flat design. Its key idea is recursive routing, and refers to the internal nodes in the hierarchy as domains. The domains in upper layers merge their children in lower layers. It looks like the DNS system. The Canon principle can be applied to many different DHTs. For example, Canon can transform Chord into its Canonical version which is called Crescendo. Upper Crescendo rings are obtained by merging lower Chord rings or Crescendo rings. When two rings are merged, nodes keep original links but add some new links.

\section{SIMULATION}

We use four types of file distributions to simulate the file distribution in an overlay network, namely, uniform distributions, dichotomy distributions, Zipf distributions, and Zipf-Mandelbrot distributions. Differences between these four file distributions are as follows. When using a uniform distribution, it means that files or objects to be shared have the same probability to be put into the groups in $\mathrm{P} 2 \mathrm{P}$ networks. The dichotomy distribution is a phenomenon that file distributions are power-law up to a point and exponential decay beyond that point. We use it here to model that the file distribution is sharp in some items and decays quite quickly. The Zipf distribution states that given some corpus of natural language utterances, the frequency of any word is inversely proportional to its rank in the frequency table. Thus, the most frequent word will occur twice as much as the second most frequent word; the second most frequent word occurs twice as much as the fourth most frequent word; etc. This law is used to refer to anything about power law probability distribution. Zipf's law states that for a population of $N$ elements, the frequency of $k$-th elements, $f(k ; s, N)$, is given by:

$$
f(k ; s, N)=\frac{1 / k^{s}}{\sum_{n=1}^{N} 1 / n^{s}},
$$

where $N$ is the number of files, $k$ is their rank, $s$ is the value of the exponent characterizing the distribution, and we use the classic version of Zipf's law, which corresponds to $s=1$, in the simulation.

The Zipf-Mandelbrot distribution is also often used to model file distributions. Zipf-Mandelbrot is a generalized version of the Zipf distribution with one more parameter. The following equation defines the probability of receiving a $k$-th rank object in $N$ objects.

$$
\begin{gathered}
P(k)=\frac{1}{H_{N, \alpha, q} \cdot(k+q)^{\alpha}}, H_{N, \alpha, q}=\sum_{i=1}^{N} \frac{i}{(i+q)^{\alpha}}, \\
q \geq 0
\end{gathered}
$$

where $\alpha$ is a skewness factor and $q$ is a plateau factor. In Saleh and Hefeeda (2006), $\alpha$ is chosen in between $[0.4,0.7]$, and $q$ is between $[5,60] . q$ is called the plateau factor because it determines the plateau shape near the left-most part of the distribution. It is noted that the higher the value of $q$, the flatter the head of the distribution will be. When $q=0$, Zipf-Mandelbrot distribution degenerates to a Zipf distribution with a skewness factor $\alpha$. Higher values of $\alpha$ mean that the difference in ranking distribution is more pronounced. In our experiments, we set $\alpha=1$ and $q=60$ to model a distribution something in between the uniform distribution and the Zipf distribution.

We add 50000 peers in order into the network to form an overlay network, respectively, set the number of files to be 15000 , and compare the properties of locality, messages per search, the hit ratio, and the hit ratio in level 0 for the four file distributions. The locality will be modeled as an index $\delta$ that denotes the improvement ratio over the randomly connected overlay and is defined as below:

$$
\delta=1-\frac{d_{L A R O}}{d_{R C O}},
$$


Table 1 Experiment results of the LARO networks with 50000 peers

\begin{tabular}{|c|c|c|c|c|c|}
\hline & $\begin{array}{l}\text { Messages } \\
\text { per search }\end{array}$ & $\begin{array}{l}\text { Locality improvement } \\
\text { ratio }\end{array}$ & Hit ratio & Hit in Level 0 & $\begin{array}{c}\text { Response time } \\
(\mu \mathrm{s})\end{array}$ \\
\hline Uniform & 81991 & $89.2 \%$ & $95.8 \%$ & $10.3 \%$ & 304773 \\
\hline Zipf-Mandelbrot & 61528 & $89.4 \%$ & $97.5 \%$ & $13.3 \%$ & 93105 \\
\hline Dichotomy & 58 & $53.5 \%$ & $99.9 \%$ & $99.6 \%$ & 4592 \\
\hline Zipf & 24705 & $80.3 \%$ & $97.6 \%$ & $57.4 \%$ & 84939 \\
\hline
\end{tabular}

Table 2 Experiment results for the LARO Networks with 100000 peers

\begin{tabular}{|c|c|c|c|c|c|}
\hline & $\begin{array}{l}\text { Messages } \\
\text { per search }\end{array}$ & $\begin{array}{l}\text { Locality improvement } \\
\text { ratio }\end{array}$ & Hit ratio & Hit in Level 0 & $\begin{array}{c}\text { Response time } \\
(\mu \mathrm{s})\end{array}$ \\
\hline Uniform & 171235 & $89.3 \%$ & $95.1 \%$ & $11.8 \%$ & 633444 \\
\hline Zipf-Mandelbrot & 123671 & $87.2 \%$ & $97.0 \%$ & $13.6 \%$ & 385106 \\
\hline Dichotomy & 60 & $53.4 \%$ & $99.9 \%$ & $99.6 \%$ & 6902 \\
\hline Zipf & 45501 & $78.5 \%$ & $96.8 \%$ & $57.6 \%$ & 118274 \\
\hline
\end{tabular}

where $d_{\text {LARO }}$ denotes the average distance under the LARO network and $d_{R C O}$ is the average distance under the randomly connected overlay (RCO) network. The simulation results are shown In Table 1, as compared to the RCO network, the LARO network has the $\delta>80 \%$ for the uniform, the Zipf and the ZipfMandelbrot distributions, while for the dichotomy distribution the LARO network gets only about $54 \%$ improvement. The reason is that there are more duplicate files under the dichotomy distribution than the former three file distributions; therefore, a node has more chance to join a group with the same resource type number instead of considering locality, thus causing average distance to increase.

After running 10000 times, the search algorithm using modified random walk has a clear trend in showing the average number of messages per search. The trend is quite coincident with the relationship with the popularity modeling capability of the file distribution we use in overlay networks. The number of duplicate files increases when there are increasing numbers of popular objects in the network. The popularity modeling capability from high to low are the dichotomy, the Zipf, the Zipf-Mandelbrot, and the uniform distributions, respectively, and the average number of messages per search from low to high are ranked the same as above. Note that using the dichotomy distribution produces more replicate files for more popular objects, so it incurs fewer messages per search than using the other three file distributions. In this case, we can almost find the queried file in level 0 as can be seen from the fact that the number of messages per search is far below the other three distributions.

In the proposed two-level random walk search algorithm as shown in Fig. 4, we make queries for the required file in groups of level 0 with the same resource type number. If the file is not found in level 0 , we search in groups of level 1 with different resource type numbers. As a result, the search time and messages per search can be reduced if we can find the needed file directly in level 0 instead of level 1. From Table 1, almost all files can be found in level 0 when using the dichotomy distribution; $60 \%$ of files can be found in level 0 when using the Zipf distribution. Although only $10.23 \%$ and $13.23 \%$ files can be found in level 0 for the uniform distribution and the Zipf-Mandelbrot distribution, respectively, more than $95 \%$ of files can be found in level 1 when using either one of the two distributions.

In addition to simulating the LARO network with 50000 peers, we also simulated LARO with 100000 peers in order to compare all kinds of simulation results after increasing the size of the overlay network. From Table 1 to Table 2, we can see that even though the size of the overlay network increases, the same results about locality, the messages per search, the hit ratio, and the hit ratio in level 0 are obtained; however, the more peers in the overlay network, the more messages per search are incurred for a query.

To compare the performance of the LARO network with that of the mOverlay network, we redo the same experiments for the mOverlay network. The results are shown in Table 3 and Table 4. To easily compare the results of Table 1 and Table 3, we present the charts shown in Fig. 6 to Fig. 8 for messages per search, the locality improvement ratio, and hit ratio, respectively.

In Fig. 5, we observe that LARO spends much fewer messages per search than mOverlay for all file distributions. It is clearly that the LARO network for the dichotomy distribution incurs many fewer messages per search than all the others. The results 
Table 3 Experiment results for the mOverlay network with 50000 peers

\begin{tabular}{|c|c|c|c|c|c|}
\hline & $\begin{array}{l}\text { Messages } \\
\text { per search }\end{array}$ & $\begin{array}{l}\text { Locality improvement } \\
\text { ratio }\end{array}$ & Hit ratio & Hit in Level 0 & $\begin{array}{c}\text { Response time } \\
(\mu \mathrm{s})\end{array}$ \\
\hline Uniform & 94505 & $90.2 \%$ & $95.7 \%$ & - & 415218 \\
\hline Zipf-Mandelbrot & 75583 & $90.2 \%$ & $97.5 \%$ & - & 160850 \\
\hline Dichotomy & 223 & $90.2 \%$ & $99.9 \%$ & - & 13109 \\
\hline Zipf & 38147 & $90.3 \%$ & $98.5 \%$ & - & 153617 \\
\hline
\end{tabular}

Table 4 Experiment results for the mOverlay networks with 100000 peers.

\begin{tabular}{|c|c|c|c|c|c|}
\hline & $\begin{array}{l}\text { Messages } \\
\text { per search }\end{array}$ & $\begin{array}{c}\text { Locality improvement } \\
\text { ratio }\end{array}$ & Hit ratio & Hit in Level 0 & $\begin{array}{c}\text { Response time } \\
(\mu \mathrm{s})\end{array}$ \\
\hline Uniform & 332962 & $90.2 \%$ & $95.9 \%$ & - & 802241 \\
\hline Zipf-Mandelbrot & 206830 & $90.3 \%$ & $97.3 \%$ & - & 470730 \\
\hline Dichotomy & 312 & $90.2 \%$ & $99.9 \%$ & - & 34677 \\
\hline Zipf & 91218 & $90.4 \%$ & $98.4 \%$ & - & 223454 \\
\hline
\end{tabular}

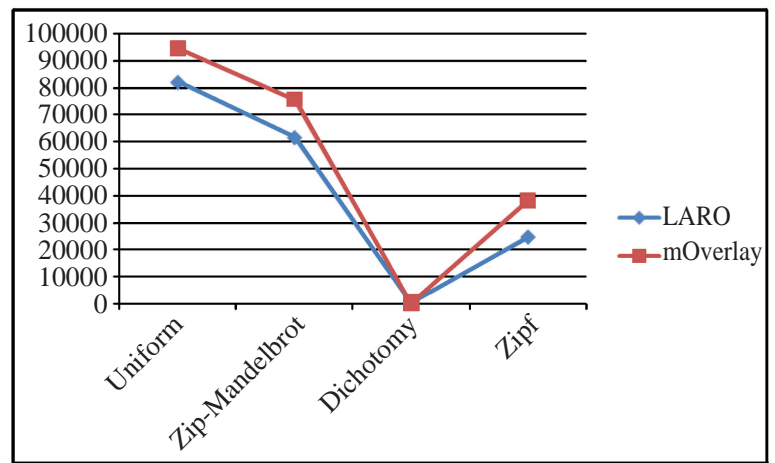

Fig. 5 Comparison of messages per search between mOverlay and LARO networks

are closely related to the sharp popularity modeled with the dichotomy distribution. In this case, there exist a lot of replications in the LARO network for the popular objects. It is noted that the Dichotomy distribution describes an extreme case that many objects fall into the same popular groups so that the grouping is dominated by the resource criteria, making the locality conditions hardly been considered in the join process.

As for the hit ratio, Fig. 6 shows that all methods can achieve above $95 \%$. However, it is interesting to note that the mOverlay network has a little bit higher hit ratio than the LARO network. The result may be due to the fact that groups of LARO are larger than those of mOverlay, thus being more difficult to search for an object in the LARO network under the same search parameters. In turn, the result of a larger group size of the LARO network may come from the fact that the locating process of the LARO network considers the resource types first and the process terminates once the resource types are matched.

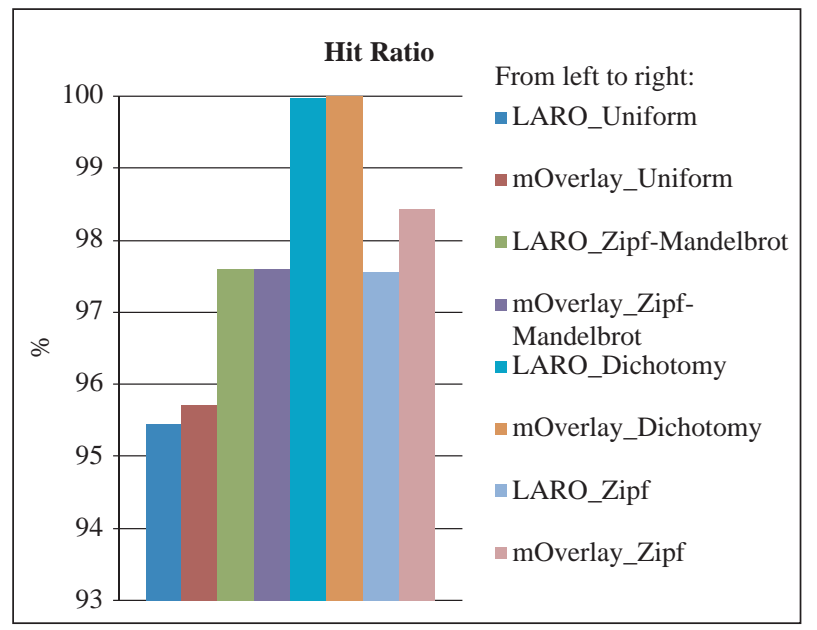

Fig. 6 Comparison of hit ratios between the LARO and the mOverlay network for different file distributions

Figure 7 shows the locality improvement ratios of the LARO and mOverlay networks with respect to the RCO network. The locality improvement ratios of the LARO networks for all file distributions are above $80 \%$ except the dichotomy distribution; the locality improvement ratios of the mOverlay network for all file distributions are about $90 \%$ because the mOverlay network concerns only locality and file distributions have no effect on it. It is interesting to note that for the uniform and the Zipf-Mandelbrot distributions the LARO networks can achieve almost $90 \%$ of locality improvement ratios, which is almost the same as mOverlay networks do. The result is explainable since the locating process of LARO network considers resource types first and then the locality. The resultant network is mainly due to the factor of locality when the resource types are nearly uniformly 


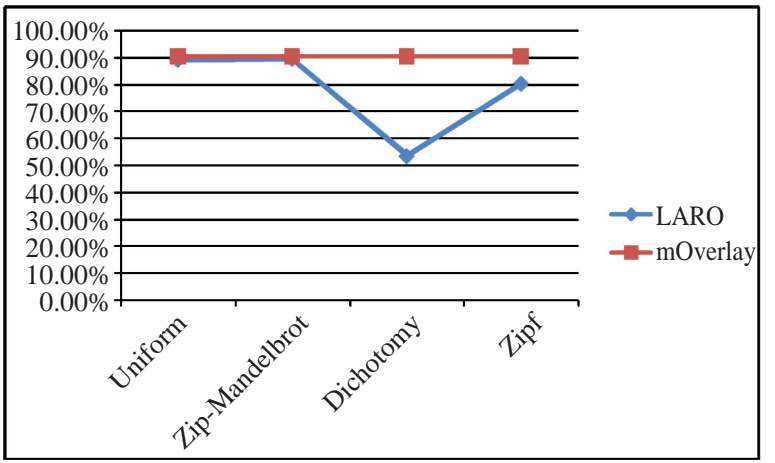

Fig. 7 Improvement of average distance between two peers for both LARO and mOverlay networks with respect to randomly connected overlay

distributed.

We are curious to know the average node degrees of the LARO network and the mOverlay degree. Fig. 8 and Fig. 9 show the average node degrees and the response time, respectively, for the LARO and mOverlay networks with 50000 peers. The average node degree reflects the number of closely related objects or duplicate files. Different types of file distributions for the LARO network cause different numbers of replicate files. The result shows that the average node degrees are $8.62,6.82,6.26$, and 6.23 for the dichotomy, Zipf, Zipf-Mandelbrot, and uniform distributions, respectively. This is quite coincident with the concepts implied by the file distributions. Average node degree for the mOverlay network is 4.4 for all distributions because it is not aware of resource types when constructing an overlay network. The results of the response time are also consistent with the observation of the number of messages, where we can see that the more pronounced the popularity of a distribution is, the quicker the query will be responded to.

\section{CONCLUSION}

We have considered both the locality and the resource awareness in constructing peer-to-peer overlay networks. We define the resource awareness as the knowledge of resource types. It is interesting to note that the resource types themselves present the properties of fractals both in coarse and fine classifications of resources. The proposed overlay network is a two-level scheme, called LARO networks, with the concept of grouping by ordered factors of locality clustering, interest groups, and data replication. The modified random walk search algorithm can achieve a high query performance in the proposed LARO networks. Simulation results show that our algorithm is better than the mOverlay network in the number of messages per search while it maintains

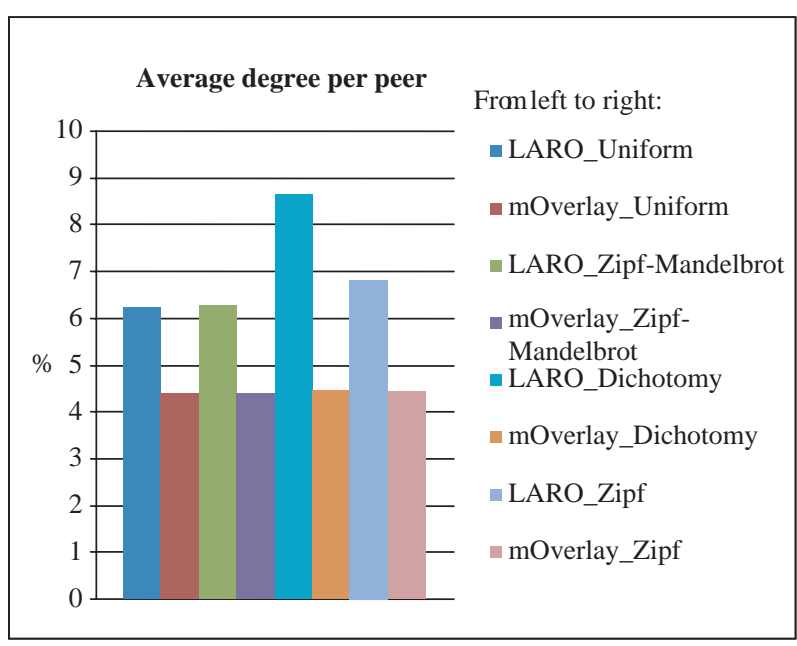

Fig. 8 Average degree of peers of the LARO and mOverlay networks for different file distributions

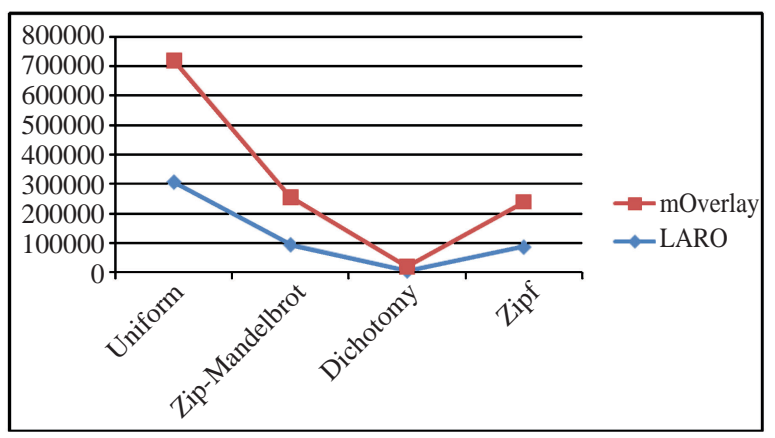

Fig. 9 Average response time $(\mu \mathrm{s})$ per query of the LARO and mOverlay networks for different file distributions

almost the same hit ratio and comes with competitive locality properties.

\section{NOMENCLATURE}

$d \quad$ average distance in hops

$D_{\text {in }} \quad$ average intra-group distances of an mOverlay

$D_{\text {ou }}$ average inter-group distances of an mOverlay

$D_{i} \quad$ average intra-group distances of an LARO network

$D_{o} \quad$ average inter-group distances of an LARO network

$\bar{D} \quad$ the average distance under LARO networks

$D_{r c o}$ the average distance of a randomly connected overlay

$\delta \quad$ the improvement ratio

$N \quad$ total nodes or groups of a network

$M \quad$ average number of neighbors

\section{ACKNOWLEDGEMENT}

The work is partially supported by a research 
grant from National Science Council, Taiwan under the contract number NSC 95-2221-E-002 -100 -MY3.

\section{REFERENCES}

Banerjee, S., Bhattacharjee, B., and Kommareddy, C., 2002, "Scalable Application Layer Multicast," Proceedings of ACM SIGCOMM, August 19-23, 2002, Pittsburgh, Pennsylvania, USA. pp. 205217.

Bestavros, A., and Jin, S., 2003, "OSMOSIS: Scalable Delivery of Real-Time Streaming Media in Ad-Hoc Overlay Networks," Proceedings of the $23 r d$ International Conference on Distributed Computing Systems Workshops, IEEE Computer Society, Vienna, Austria, pp. 214-219.

Chen, W. -T., Chao, C. -H., and Chiang, J. -L., 2006, "An Interest-Based Architecture for Peer-to-Peer Network Systems," Proceedings of the 20th International Conference on Advanced Information Networking and Applications (AINA'06), Vienna, Austria, Vol. 1, pp. 707-712.

Chu, Y. -H., Rao, S. G., and Zhang, H., 2000, “A Case for End System Multicast," Proceedings of ACM SIGMETRICS, pp. 1-12.

Cohen, E., and Shenker, S., 2002, "Replication Strategies in Unstructured Peer-to-Peer Networks," SIGCOMM Computer Communication Review, Vol. 32 No. 4, pp. 177-190.

Cramer, C., Kutzner, K., and Fuhrmann, T., 2004, "Bootstrapping Locality-Aware P2p Networks," Proceedings of IEEE International Conference on Networks, Singapore, pp. 357-361.

Doval, D., and O'Mahony, D., 2003, "Overlay Networks: A Scalable Alternative for P2P," IEEE Internet Computing, Vol. 7 No. 4, pp. 79-82.

Ganesan, P., Gummadi, K., and Garcia-Molina, H., 2004, "Canon in G major: designing DHTs with hierarchical structure," Proceedings of International Conference on Distributed Computing Systems, Hachioji, Tokyo, Japan, pp. 263-272.

Hsiao, H. -C., and Liao, H., 2006, "The Peering Problem in Tree-Based Master/Worker Overlays," Proceedings of International Grid Pervasive Computing Conference, Taichung, Taiwan, pp. 83-92.

Kobayashi, H., Takizawa, H., Inaba, T., Takizawa, Y., 2005, "A Self-Organizing Overlay Network to Exploit the Locality of Interests for Effective Resource Discovery in P2P Systems," Proceedings of the The 2005 Symposium on Applications and the Internet, Trento, Italy, pp. 246-255.

Liu, Y., Zhuang, Z., Xiao, L., and Ni, L. M., 2004, "A Distributed Approach to Solving Overlay Mismatching Problem," Proceedings of International Conference on Distributed Computing Systems,
Hachioji, Tokyo, Japan pp. 132-139.

Lv, Q., Cao, P., Cohen, E., Li, K., and Shenker, S., 2002, "Search and replication in unstructured Peer-to-Peer networks," Proceedings of the 16th international conference on Supercomputing, ACM, New York, USA, pp. 84-95.

Lv, Q., Ratnasamy, S., Shenker, S., 2002, "Can Heterogeneity Make Gnutella Scalable?" The First International Workshop on Peer-to-Peer Systems, Springer-Verlag, Cambridge, MA, USA, pp. 94103.

Patro, S., Hu, Y. C., 2003, “Transparent Query Caching in Peer-to-Peer Overlay Networks," Proceedings of the 17th International Symposium on Parallel and Distributed Processing, IEEE Computer Society, Nice, France, pp. 10.

Ratnasamy, S., Francis, P., Handley, M., Karp, R., Shenker, S., 2001, "A Scalable Content-Addressable Network," Proceedings of the 2001 Conference on Applications, Technologies, Architectures, and Protocols for Computer Communications, ACM, San Diego, California, USA, pp. 161-172.

Ratnasamy, S., Handley, M., Karp, R., and Shenker, S., 2002, "Topologically-aware Overlay Construction and Server Selection," Proceedings INFOCOM 2002. Twenty-First Annual Joint Conference of the IEEE Computer and Communications Societies. IEEE, New York, NY USA, vol. 3, pp. 1190-1199.

Rowstron, A., and Drusche1, P., 2001, "Pastry: Scalable, Distributed Object Location and Routing for Large-scale Peer-to-Peer Systems" In IFIP/ ACM International Conference on Distributed Systems Platforms (Middleware), Heidelberg, Germany, pp. 329-350.

Saleh, O., and Hefeeda, M., 2006, "Modeling and Caching of Peer-to-Peer Traffic," Proceedings of International Conference on Network Protocols, Santa Barbara, California, USA, pp. 249-258.

Shamsi, J., Brockmeyer, M., and Abebe, L., 2005, "TACON: Tactical Construction of Overlay Networks," Proceedings of Global Telecommunications Conference, GLOBECOM '05. IEEE, St. Louis, MO, USA, pp. 926-931.

Shin, K., Lee, S., Lim, G., Yoon, H., and Ma, J. S., 2002, "Grapes: Topology-Based Hierarchical Virtual Network for Peer-to-Peer Lookup Services," International Conference on Parallel Processing Workshops (ICPPW'02), Vancouver, British Columbia, Canada, pp. 159-164.

Si, W., and Li, M., 2005, "On the Connectedness of Peer-to-Peer Overlay Networks," Proceedings of 11th International Conference on Parallel and Distributed Systems, Fukuoka, Japan, pp. 474480.

Stoica, I., Morris, R., Karger, D., Kaashoek, M. F., and 
Balakrishnan, H., 2001, "Chord: A Scalable Peerto-Peer Lookup Service for Internet Applications." Proceedings of the 2001 Conference on Applications, Technologies, Architectures, And Protocols for Computer Communications, ACM, San Diego, California, USA, pp. 149-160.

Sun, Y., Sun, L., Huang, X., and Lin, Y., 2006, "Resource Discovery in Locality-Aware Group-Based Semantic Overlay of Peer-to-Peer Networks," Proceedings of the 1st International Conference on Scalable Information Systems, ACM, Hong Kong, Article No. 44.

Wang, J. Z., and Vanninen, M. A., 2004, “A Novel Self-Configuration Mechanism for Heterogeneous P2P Networks," Proceedings of IEEE/WIC/ACM International Conference the Intelligent Agent Technology, IEEE, Beijing, China, pp. 281-287.

Xiao, L., Liu, Y., and Ni, L. M., 2005, "Improving Unstructured Peer-to-Peer Systems by Adaptive Connection Establishment," IEEE Transactions on Computers, Vol. 54 No. 9, pp. 1091-1103.

Xu, Z., Min, R., Hu, Y., 2003, "HIERAS: a DHT Based Hierarchical P2P Routing Algorithm," Proceedings of International Conference on Parallel Processing, Kaohsiung, Taiwan, pp. 187-194.

Xu, Z., Tang, C., Zhang, Z., 2003, "Building Topology-Aware Overlays Using Global Soft-State," Proceedings of the 23rd International Conference on Distributed Computing Systems, IEEE, Providence, Rhode Island USA, pp. 500-508.

Yang, B., and Garcia-Molina, H., 2002 "Efficient
Search in Peer-to-Peer Networks," Proceedings International Conference on Distributed Computing Systems, Vienna, Austria, pp. 5-15.

Zhang, B., Jamin, S., and Zhang, L., 2002, "Host multicast: A framework for delivering multicast to end users," Proceedings of IEEE INFOCOM, New York, NY, USA, Vol. 3, pp. 1366-1375.

Zhang, J., Liu, L., and Pu, C., 2005, "Constructing a Proximity-Aware Power Law Overlay Network," Proceedings of Global Telecommunications Conference, GLOBECOM '05. IEEE, St. Louis, MO, USA, pp.636-640.

Zhang, X. Y., Song, G., Zhang, Q., Zhu, W., Gao, L., and Zhang, Z., 2004, "Measurement-Based Construction of Locality-Aware Overlay Networks," IEEE International Conference on Communications, Paris, France, pp. 1401-1405.

Zhang, X. Y., Zhang, Q., Zhang, Z., Song, G., and Zhu, W., 2004, "A Construction of Locality-Aware Overlay Network: mOverlay and Its Performance," IEEE Journal Selected Areas in Communications, Vol. 22, No. 1, pp. 18-28.

Zhao, B. Y., Kubiatowicz, J., and Joseph, A. D., 2001, "Tapestry: An Infrastructure for Fault-tolerant Wide-area Location and Routing," Technical Report, UCB/CSD-01-1141, 28 pages, University of California at Berkeley, CA, USA.

Manuscript Received: Apr. 10, 2008

Revision Received: Aug. 24, 2008 and Accepted: Sep. 24, 2008 